\title{
CONSUMO DE ALIMENTOS IMPRÓPRIOS POR CRIANÇAS MENORES DE DOIS ANOS E SUAS POSSÍVEIS CONSEQUENCIAS
}

Autor: Fabrícia de Souza FERREIRA*

* Nutricionista, pós graduanda em Nutrição Clínica, fabriciasouzaferreira@gmail.com.

Recebido em: 10/11/2014 - Aprovado em: 04/06/2015 - Disponibilizado em: 15/07/2015

\begin{abstract}
RESUMO: A alimentação nos primeiros anos de vida está fortemente ligada à saúde, crescimento e desenvolvimento infantil, de modo que uma amamentação e alimentação adequada repercutem positivamente por toda a vida. Objetivouse avaliar a ingestão de alimentos impróprios por crianças menores de dois anos residentes no município de João Pessoa/PB. Trata-se de um estudo transversal quantitativo. Em relação à idade materna, observou-se que a idade predominante foi entre a faixa etária de 20 a 29 anos (57\%); no que diz respeito ao perfil socioeconômico, observou-se que a maioria das mães apresentou ensino médio completo (48\%); $68 \%$ moram com o companheiro; $51 \%$ afirmaram ser do lar e $57 \%$ são primíparas. Quanto à renda familiar, $46 \%$ das mães relataram receber até um salário mínimo. Com relação ao aleitamento materno exclusivo, $71 \%$ interromperam precocemente e sobre o aleitamento complementar, $44 \%$ tiveram o desmame total antes de 12 meses. Em relação aos alimentos impróprios, observou-se que o leite de vaca e os derivados lácteos foram os alimentos mais utilizados apresentando $79 \%$ e $71 \%$ respectivamente, seguidos pelos alimentos doces (70\%), açúcar refinado (68\%) e sopas/macarrões instantâneos (63\%). Foi observada uma alta frequência no abandono do aleitamento exclusivo precocemente e do tempo do aleitamento materno, além de uma elevada prevalência de consumos de alimentos impróprios para menores de dois anos.
\end{abstract}

Palavras-chave: Aleitamento. Alimentação Complementar. Aditivos. Desmame precoce. Lactentes

\begin{abstract}
The feed early in life is strongly linked to children health, growth and development so that a breastfeeding and proper nutrition reverberate positively throughout their lives. To evaluate the intake of inappropriate foods for children under two years old living in the city of João Pessoa/Pb. It is a quantitative cross-sectional study. Regarding maternal age, it was observed that the predominant age was between the age group 20 to 29 years old (57\%); About the socioeconomic profile, it was observed that the majority of the mothers had completed the high school (48\%); 68\% live with a partner; $51 \%$ said being housewives and 57\% are primiparous. Regarding the family income, $46 \%$ of the mothers reported receiving up to a minimum wage. With regard to exclusive breastfeeding, $71 \%$ stopped it early end On supplementary feeding, $44 \%$ had full weaming before 12 months. In relation to unsuitable products, it was observed that the cow milk and milk derivatives have been the most used foods, having $79 \%$ and $71 \%$ respectively, followed by sweet foods (70\%), refined sugar $(68 \%)$ and soups/instant noodles $(63 \%)$. It was observed a high rate of abandon of the exclusive breastfeeding and the time of maternal breastfeeding, in addition to a high prevalence of consumption of inappropriate foods for children under two years old.
\end{abstract}

Keywords: Breastfeeding. Complementary feeding. Additives. Early Weaning. Infats.

\section{INTRODUÇÃO}

A alimentação nos primeiros anos de vida está fortemente ligada à nutrição, saúde, desenvolvimento e crescimento infantil de modo que uma alimentação adequada é essencial para auxiliar no incremento da atividade vital da criança e têm repercussões ao longo de toda a sua vida (BRITO et al.,
2014; CORREIA; PEREIRA; BRITO, 2013; TOLONI et al., 2011).

O aleitamento materno é um extraordinário componente da alimentação infantil por ser o único alimento apto a suprir todas as necessidades nutricionais do bebê devendo ser o único alimento administrado nos seis primeiros meses de vida, com adição de alimentos complementares e continuação da 
amamentação a partir de então e até os dois anos de vida ou mais (BRASIL et al., 2012).

A prática alimentar nos dois primeiros anos de vida tem uma importante influência na concepção dos hábitos alimentares da criança e é diretamente induzida pelo ambiente familiar, este, quando desfavorável propicia condições que induzem ao incremento de morbimortalidade e distúrbios alimentares que, uma vez adquiridos, são de difícil reversão. Além disso, alterações, carências ou desajustes em fases precoces da vida, como na infância, pode resultar em consequências em longo prazo para as funções fisiológicas (MARQUES et al., 2013; OLIVEIRA; BOSCO, 2009; MONTE; GIUGLIANI, 2004).

O presente trabalho objetivou avaliar a ingestão de alimentos impróprios por crianças menores de dois anos residentes no município de João Pessoa-PB e as possíveis consequências.

\section{METODOLOGIA}

Trata-se de um estudo transversal, realizado na cidade de João Pessoa/ PB. Participaram desse estudo, crianças com idade entre seis meses e menores de dois anos, de ambos os gêneros, residentes na cidade de João Pessoa/PB.

As crianças foram selecionadas através de uma amostra não probabilística de conveniência. Os dados foram coletados em Unidades de Saúde e hospitais de referência infantil da cidade entre os meses de Novembro de 2013 a Janeiro de 2014.

A coleta foi realizada através de uma entrevista estruturada onde foram inquiridas informações sobre as variáveis socioeconômica da mãe da criança; dados sobre os hábitos alimentares dos lactentes como o uso, a frequência e a idade da introdução de alimentos como: de leite de vaca, mel, amendoim, crustáceos, iogurte ou bebida láctea, suco industrializado e artificial em pó, café, açúcar de adição, embutidos, doces (guloseimas, chocolate...), refrigerantes, sopa e macarrão instantâneos, biscoitos recheados, salgadinhos de pacote, frituras em imersão, enlatados e prontos congelados.

Os dados foram registrados em uma planilha do Microsoft Office Excel 2010 e posteriormente analisados.

\section{RESULTADOS E DISCUSSÃO}

Participaram da pesquisa 63 crianças entre 6 a 23 meses e 29 dias, sendo $43 \%$ com a faixa etária de 6 a 12 meses e $57 \%$ acima de 12 meses. Em relação à idade materna, a idade predominante foi entre a faixa etária de 20 a 29 anos, que correspondeu a $57 \%$ das entrevistadas.

No que diz respeito ao perfil socioeconômico da população estudada, observou-se que a maioria das mães entrevistadas apresentou ensino médio completo como nível de escolaridade, representando $48 \%$ da amostra; $68 \%$ moram com o companheiro; $51 \%$ das mães afirmaram 
ser do lar e $57 \%$ são primíparas. Quanto à renda familiar $46 \%$ das mães relataram receber até um salário mínimo (Tabela 1).

Tabela 1 - Distribuição dos dados pessoais e das variáveis socioeconômico das mães entrevistadas em João Pessoa-PB, Brasil, 2014.

\begin{tabular}{|c|c|c|}
\hline Variáveis & $\mathbf{N}^{\mathbf{0}}$ & $\%$ \\
\hline \multicolumn{3}{|l|}{ Escolaridade da mãe } \\
\hline Superior completo & 2 & 3 \\
\hline Superior incompleto & 9 & 14 \\
\hline Médio completo & 30 & 48 \\
\hline Médio incompleto & 7 & 11 \\
\hline Fundamental completo & 8 & 13 \\
\hline Fundamental incompleto & 7 & 11 \\
\hline \multicolumn{3}{|l|}{ Estado Civil } \\
\hline Casada & 43 & 68 \\
\hline Solteira & 20 & 32 \\
\hline \multicolumn{3}{|l|}{ Idade } \\
\hline$<19$ & 6 & 10 \\
\hline 2029 & 36 & 57 \\
\hline$>30$ & 21 & 33 \\
\hline \multicolumn{3}{|l|}{ Ocupação } \\
\hline Do lar & 32 & 51 \\
\hline Trabalha/estuda & 31 & 49 \\
\hline \multicolumn{3}{|l|}{ Renda Familiar } \\
\hline Até 1 salário & 29 & 46 \\
\hline De 1 a 3 salários & 26 & 41 \\
\hline$>3$ salários & 8 & 13 \\
\hline
\end{tabular}

Fonte: João Pessoa (PB); Pesquisa de Campo, 2013.

Com relação ao aleitamento materno exclusivo (AME), $71 \%$ crianças menores de dois anos avaliadas, tiveram o AME interrompido precocemente, sendo que $44 \%$ introduziram algum tipo de alimentação antes do quarto mês. No que diz respeito ao aleitamento materno, $44 \%$ tiveram o desmame total antes de 12 meses, sendo que 17\% abandonaram o aleitamento antes dos seis meses completos (Tabela 2).
Tabela 2: Duração do aleitamento materno exclusivo e abandono total da amamentação. João Pessoa-PB, Brasil, 2014.

\begin{tabular}{lccc}
\hline \multicolumn{1}{c}{ Variáveis } & $\mathbf{N}^{\mathbf{0}}$ & $\mathbf{\%}$ \\
\hline AME & Até $6^{\mathbf{0}}$ mês & 18 & 29 \\
Até $5^{\mathbf{0}}$ mês & 6 & 10 \\
Até $4^{\mathbf{o}}$ mês & 11 & 17 \\
Até $3^{\mathbf{0}}$ mês & 14 & 22 \\
Até 2 meses & 5 & 8 \\
< 1 mês ou nunca & 9 & 14 \\
Aleitamento Materno & & \\
Ainda mama & 29 & 46 \\
> 12 meses & 6 & 10 \\
De 6 a 12 meses & 17 & 27 \\
$<6$ meses & 11 & 17 \\
\hline Fonte: João Pessoa (PB); Pesquisa de Campo, 2013.
\end{tabular}

Os valores encontrados para a interrupção do AME são semelhantes a outro estudo publicado no final de 2013 realizado em Campina Grande-PB onde $67,7 \%$ das crianças introduziram outros alimentos e/ou líquidos antes do tempo preconizado, sendo que $44 \%$ interromperam o $\mathrm{AME}$ antes de um mês (ARAÚJO, 2013).

Pesquisa comprova que o abandono precoce do aleitamento exclusivo predispõe ao aparecimento de obesidades e doenças crônicas (SIMON; SOUZA; SOUZA, 2009) Além de propiciar o aparecimento de morbidades como doenças diarreicas, infecciosas e do trato respiratório (ARAÚJO et al., 2006; BERNARDI, 2008).

Em relação ao consumo de alimentos inadequados, a Tabela 3 lista a prevalência da ingestão desses alimentos oferecidos às crianças antes e após os seis meses de idade com a frequência de consumo de no mínimo uma vez ao mês. 
Tabela 4: Prevalência de consumo de alimentos não recomendados com frequência mínima de uma vez mensal por crianças menores de dois anos residentes no município de João Pessoa -PB, Brasil, 2014.

\begin{tabular}{|c|c|c|c|}
\hline \multirow{2}{*}{ ALIMENTO } & \multicolumn{3}{|c|}{ CONSUMO } \\
\hline & $<6$ Meses & $>6$ Meses & Total \\
\hline Leite de vaca & $41 \%$ & $38 \%$ & $79 \%$ \\
\hline Iogurte ou bebida láctea & $35 \%$ & $37 \%$ & $71 \%$ \\
\hline $\begin{array}{l}\text { Doces (bala, chocolate, } \\
\text { pirulito, etc) }\end{array}$ & $14 \%$ & $56 \%$ & $70 \%$ \\
\hline Açúcar de adição & $40 \%$ & $29 \%$ & $68 \%$ \\
\hline $\begin{array}{l}\text { Sopa/macarrão } \\
\text { instantâneos }\end{array}$ & $25 \%$ & $38 \%$ & $63 \%$ \\
\hline $\begin{array}{l}\text { Suco industrializado } \\
\text { líquido ou em pó }\end{array}$ & $16 \%$ & $41 \%$ & $57 \%$ \\
\hline Biscoito recheado & $0 \%$ & $56 \%$ & $56 \%$ \\
\hline Frituras & $0 \%$ & $46 \%$ & $46 \%$ \\
\hline Embutidos & $5 \%$ & $37 \%$ & $41 \%$ \\
\hline Salgadinho de pacote & $2 \%$ & $37 \%$ & $38 \%$ \\
\hline Refrigerante & $0 \%$ & $38 \%$ & $38 \%$ \\
\hline Enlatados & $0 \%$ & $30 \%$ & $30 \%$ \\
\hline Amendoim ou castanha & $2 \%$ & $21 \%$ & $22 \%$ \\
\hline Mel & $6 \%$ & $14 \%$ & $21 \%$ \\
\hline Café & $0 \%$ & $19 \%$ & $19 \%$ \\
\hline Crustáceos & $0 \%$ & $14 \%$ & $14 \%$ \\
\hline Congelados prontos & $0 \%$ & $13 \%$ & $13 \%$ \\
\hline
\end{tabular}

Fonte: João Pessoa (PB); Pesquisa de Campo, 2013.

Quanto à introdução de alimentos complementares, os resultados mostraram que há uma grande prevalência na introdução precoce de alimentos não recomendados, em desacordo com as recomendações dos órgãos nacionais e internacionais de saúde que preconiza que não seja introduzido nenhum tipo de alimento antes dos seis meses de idade (BRASIL, 2009a; WHO, 2001).

O leite de vaca e os derivados lácteos, como iogurte e bebida láctea, foram os alimentos mais consumidos pelas crianças avaliadas, apresentando $79 \%$ e $71 \%$ respectivamente, seguidos pelos alimentos doces $(70 \%)$ e o açúcar refinado (68\%) adicionado às preparações caseiras.

Em relação ao consumo do leite de vaca, observou-se que $41 \%$ das crianças consumiram esse alimento antes dos seis meses de idade, sendo que $38 \%$ consumiram com uma frequência diária.

Pesquisa realizada em Curitiba, Recife e São Paulo, mostraram um elevado consumo de leite de vaca em crianças menores de um ano (CAETANO et al., 2010) e em BelémPará, um estudo conduzido com lactentes também mostrou um grande percentual de introdução de leite de vaca antes do sexto mês de vida (MARQUES et al., 2013).

O uso precoce de leite de vaca, principalmente antes dos 12 meses é contra indicado por desencadear alergias alimentares, sobrecarga renal e imunológica, doenças crônicas como obesidade e diabetes mellitus, além de interferir negativamente na absorção do ferro podendo desencadear anemia ferropriva. A cada mês de uso contínuo do leite de vaca há uma queda de $0,2 \mathrm{~g} / \mathrm{dL}$ nos níveis de hemoglobina, podendo ocasionar pequenas hemorragias e sequelas no desenvolvimento cognitivo e emocional da criança (CAETANO et al., 2010; MARQUES, et al., 2013; OLIVEIRA; OSÓRIO, 2005).

$\mathrm{O}$ consumo precoce de produtos como iogurte e bebida láctea também foram observados em pesquisa realizada em Teresina onde o percentual desses alimentos atingiram $76,47 \%$ na faixa de 7 a 9 meses, 
permanecendo nesse patamar na idade acima de doze meses (CARDOSO, et al., 2013).

Estes produtos, assim como o leite de vaca, não devem ser ingeridos nessa idade por interferirem na absorção de nutrientes importantes existentes no leite materno como o ferro e o zinco (HEITOR; RODRIGUES; SANTIAGO, 2011). Além do mais, as bebidas lácteas e o iogurte apresentam uma grande quantidade de corantes e, segundo a FAO/WHO (1995), corantes e outros aditivos não devem ser ingeridos por crianças menores de um ano, como rege o Codex Alimentarius (FAO/WHO, 1995 apud POLÔNIO, 2010), sendo que $35 \%$ das mães entrevistadas informaram que introduziram iogurte ou bebida láctea na alimentação dos seus filhos antes dos seis meses de idade.

Outro consumo considerado elevado foram os doces e o açúcar de adição, representando $70 \%$ e $68 \%$ respectivamente da população estudada, sendo que $40 \%$ consumiram o açúcar na adição de preparações antes dos sexto mês vida. Resultados semelhantes foram encontrados (ALVES et al., 2012; BERNARDI; JORDÃO; BARROS FILHO, 2009; VITOLO et al., 2013).

Esses alimentos contribuem para o acréscimo da densidade energética na dieta, possui índice glicêmico elevado e é isento de outros nutrientes importantes para a criança, levando a criança à formação de maus hábitos alimentares e ao risco de carências nutricionais e morbidades na idade adulta (DIAS, 2013; HEITOR; RODRIGUES; SANTIAGO, 2011). O consumo de alimentos instantâneos, como sopas e macarrões, e o consumo de sucos industrializados líquidos e em pó também foram muito citados no consumo dos lactentes desse estudo, mas o que chamou atenção foi o consumo considerável desses alimentos antes dos seis meses de idade, sendo de $25 \%$ para alimentos instantâneos e $16 \%$ para os sucos industrializados.

Um estudo observou o consumo desses alimentos sendo introduzidos a partir do quarto mês de vida e mostrou que $92 \%$ das crianças acima de seis meses consumiram esses alimentos em alta frequência devido à facilidade de preparo e consumo (HEITOR; RODRIGUES; SANTIAGO, 2011). Toloni et al (2011), observou que o consumo, tanto de sopas e macarrões instantâneos como os sucos artificiais em pó e os industrializados foram introduzidos antes dos 3 meses de idade.

Os sucos industrializados e os artificiais, além de conterem quantidades absurdas de corantes e açúcares, contém, assim como os alimentos instantâneos, glutamato monossódico, um flavorizante altamente tóxico ao sistema nervoso central podendo causar sérios transtornos como hiperatividade, autismo, déficit de desenvolvimento e atenção, depressão e distúrbios como obesidade e diabetes, não devendo ser oferecido a crianças menores de 2 anos devido à imaturidade 
cognitiva dos mesmos (HOCAYEN, 2012; CARVALHO et al., 2011).

Além disso, as sopas de pacotes e os macarrões instantâneos contêm grandes quantidades de sal e de etilenodiaminotetracético (EDTA), um aditivo quelante, presente em temperos de alimentos instantâneos capaz de inibir a absorção do ferro não heme, podendo acarretar anemia ferropriva (CAETANO et al., 2010; HEITOR, RODRIGUES, SANTIAGO, 2011).

Não houve introdução de biscoitos recheados e frituras antes dos seis meses de vida, contudo o consumo entre as crianças maiores de seis meses e menores de dois anos foi elevada, sendo mais de $50 \%$ do consumo para biscoitos recheados. Porém, pesquisa realizada por Toloni et al (2011) mostrou a introdução de biscoito recheado antes dos três meses de idade e de frituras por imersão a partir dos quatro meses. Outros achados mostram o consumo de $10,4 \%$ de biscoitos recheados antes do sexto mês de vida (MARZANO, 2013).

Além do excesso de açúcar e a densidade calórica assustadora, os biscoitos recheados contêm muita gordura saturada e diversos tipos de aditivos, fatores de risco para o desenvolvimento futuro morbidades. Quando consumidos precocemente, pode desencadear também hiperatividade, irritabilidade e alergias. Já as frituras, além das gorduras saturadas há a formação das gorduras trans, gordura diretamente relacionada ao aparecimento de doenças cardiovasculares. (LEVY-COSTA et al., 2005; VINHOLES; ASSUNÇÃO; NEUTZLING, 2009).

Foi relatado nessa pesquisa o consumo de embutidos e salgadinhos de pacote antes do sexto mês. Os embutidos são ricos em gorduras saturadas e aditivos, como os nitritos, conservantes tóxicos com ação carcinogênica, podendo acarretar vários tumores em longo prazo, principalmente os gastrointestinais, além de doenças cardíacas. Esse aditivo pode agir sobre a hemoglobina, em lactentes e em pessoas sensíveis e impedir o transporte normal do oxigênio (POLÔNIO, 2010).

Pesquisa realizada por Toloni et al (2011) mostrou que houve o consumo de embutidos para crianças menores de três meses $(0,4 \%)$, mesmo sendo um percentual pequeno, é um sério risco, uma vez que lactentes nessa idade podem até morrer devido à imaturidade fisiológica, incapazes de digerir essas substâncias. O comitê $\mathrm{FAO} / \mathrm{WHO}$, proibiu terminantemente o emprego de nitrito em alimentos infantis para crianças menores de três meses. $\mathrm{O}$ uso desse aditivo não é indicado antes dos dois anos de idade (FIGUEIRÓ, 2013; POLÔNIO, 2010; SEMEDO, 2009).

Seguidamente, os salgadinhos de pacote, tanto de milho quanto de batata, apresentam aditivos diversos, gorduras saturadas e gorduras trans, podendo levar ao aparecimento de processos inflamatórios e doenças crônicas. Os salgadinhos de pacotes, principalmente as batatas, apresentam teor alto 
de acrilamida, uma substância altamente cancerígena, assim como a acroleína adquirida no processo de fritura (DIAS, 2013; LEVYCOSTA et al., 2005; VINHOLES; ASSUNÇÃO; NEUTZLING, 2009).

Outros estudiosos avaliaram a prevalência do consumo de alimentos industrializados para crianças entre seis meses e um ano e observou que $23,2 \%$ consumiram embutidos antes de um ano e 40,8\% salgadinho de pacote (HEITOR, RODRIGUES, SANTIAGO, 2011).

Também foi observado o consumo de enlatados em congelados prontos nas crianças pesquisadas. Os alimentos enlatados são ricos em sulfitos, um conservante muito relacionado a reações adversas em lactentes podendo desencadear alergias, irritação gástricas, hiperatividade, diarreias e crise asmáticas, em longo prazo, pode desencadear neoplasias (FAVERO; RIBEIRO; AQUINO, 2011).

Já os prontos congelados, assim como os instantâneos, salgadinhos de pacote e os embutidos, são ricos em sódio, além de outros aditivos comuns em produtos industrializados, segundo Brasil (2010), alimentos ricos em sódio ou em sal não devem ser oferecidos para crianças menores de dois anos por ser um irritante gástrico, estimular a perda de cálcio pela urina e promover hábitos inadequados que futuramente pode vir a desencadear problemas sérios de saúde (ANVISA, 2013).

Também foi analisada, nessa pesquisa, a ingestão do uso de refrigerantes e café.
Observou-se que o consumo de refrigerantes representou $38 \%$ da amostra e o consumo de café foi de $19 \%$.

Estudo realizado em São Paulo/SP observou que o consumo inicial de refrigerante ocorreu antes dos três meses de idade e 66,7\% consumiram antes de um ano. Ainda na mesma pesquisa observou-se que o uso do café foi introduzido por alguns antes dos três meses de vida, sendo que $40,8 \%$ consumiram antes dos doze meses (TOLONI et al., 2011).

O consumo de refrigerantes e cafés pode levar a uma redução da absorção de ferro não heme e afetar adversamente a relação cálcio/fósforo por conter compostos fenólicos (flavonoides, ácidos fenólicos, polifenóis e taninos) e que também estão presentes no chocolate, chá preto e mate (HEITOR; RODRIGUES; SANTIAGO, 2011; MARQUES et al., 2013).

O consumo elevado de refrigerantes entre crianças é preocupante, além de possuírem uma enorme diversidade de corantes, eles são extremamente ácidos para o organismo infantil, podendo enfraquece os ossos por reduzir o teor de cálcio, devido ao excesso de acidulantes, como o ácido fosfórico (FLORES et al., 2013; POLÔNIO, 2010).

Em relação ao consumo de amendoim e crustáceos foi observado o consumo destes pelos lactentes participantes da pesquisa, no entanto, estes devem ser evitados em crianças menores de dois anos devido ao grande potencial alergênico. Recomenda-se que a 
introdução desses alimentos aconteça bem após o primeiro ano de idade para crustáceos e volta dos três anos de idade, pode-se introduzir o amendoim e outros alimentos oleoginosos. As manifestações clínicas podem variar de urticária leve a reações sistémicas com morte por anafilaxia. Crianças com abandono precoce do aleitamento e casos na família devem evitar esses alimentos mesmo após os dois anos de idade (TEIXEIRA, 2010; PEREIRA; MOURA; CONSTANT, 2008).

Já o mel deve ser evitado antes do primeiro ano de vida, pois pode oferecer risco de contaminação com o Clostridium botulinum, microorganismo causador do botulismo (PILLA, 2009).

A introdução inadequada e precoce de alimentos complementares está diretamente associada ao atraso de desenvolvimento e ao aparecimento de morbidades infantis. Crianças menores de dois anos estão em desenvolvimento físico e psicológico e a alimentação adequada juntamente ao aleitamento até o sexto mês é fundamental para o bom desenvolvimento dessa criança.

\section{CONCLUSÃO}

Foi observada uma alta frequência no abandono do aleitamento exclusivo e do tempo do aleitamento materno, além de uma elevada prevalência de consumos de alimentos impróprios para menores de dois anos. Esse padrão inadequado de consumo alimentar pode trazer inúmeros riscos para a saúde da criança em curto e longo prazo.
Nessa perspectiva, fazem-se necessárias ações para conscientizar os pais a respeito da idade adequada e a forma correta da introdução alimentar, informando sobre os malefícios causados pela introdução precoce e imprópria de alimentos.

$\mathrm{O}$ aleitamento materno exclusivo até o sexto mês e a alimentação complementar adequada podem contribuir para a redução da incidência e mortalidade e morbidade nos próximos anos.

\section{REFERÊNCIAS}

ALVES, C.R.L. et al. Alimentação complementar em crianças no segundo ano de vida. Rev Paul Pediatr. Minas Gerais, v. 30, n. 4, p. 499-506, dez. 2012;

ANVISA. Informe técnico, 2013. Disponível em: http://s.anvisa.gov.br/wps/s/r/c86d. Acesso em: 25 jan. 2014;

ARAÚJO, M.F.M. et al. Avanços na norma brasileira de comercialização de alimentos para idade infantil. Rev Saúde Pública, Brasília, v. 40, n. 3, p. 513-520, 2006;

ARAÚJO, J.P. Desmame precoce e suas causas: experiência na atenção básica de campina grande-pb. Revista da Universidade Vale do Rio Verde, Três Corações, v. 11, n. 2, p. 146-155, ago./dez. 2013; 
BERNARDI, J.L.D ; JORDÃO, R.E;

BARROS FILHO, A.A. Alimentação

complementar de lactentes em uma cidade

desenvolvida no contexto de um país em

desenvolvimento. Rev Panam Salud

Publica/Pan Am J Public Health,

[Campinas], v. 26 n.5, 2009;

BERNARDI, M. A relação entre a incidência de infecções respiratórias agudas em crianças de 0 a 2 anos e o desmame precoce como fator de risco: um estudo na secretaria municipal de saúde do município de São Miguel do Oeste - SC. 66f. 2008. Trabalho de Conclusão de Curso (Bacharelado em Enfermagem) - Universidade do Estado de Santa Catarina, Palmitos, 2008;

BRASIL, L.M.B.F et al. Introdução de alimentos complementares em lactentes/ Introduction of complementary feeding in children. Rev. para. med., v. 26, n. 1, Não paginado, jan./mar. 2012;

BRASIL, Ministério da Saúde. Saúde da criança: nutrição infantil. Aleitamento materno e alimentação complementar. Série A. Normas e Manuais Técnicos. Caderno de Atenção Básica, no 23, Brasília, DF: Ministério da Saúde, 2009a;

BRITO, R.S. et al. Conhecimento de profissionais de saúde acerca da distribuição do leite humano pasteurizado. J. res.: fundam. care. Online, [S.I], v.6, n.1, p. 261270, jan./mar. 2014;

CAETANO, M.C. et al. Alimentação complementar: práticas inadequadas em lactentes. Arch Pediatr Urug, [S.I], v. 83, n. 3, p. 226-232, 2010;

CARDOSO, E.K.S. et al. Industrialized food consumption by children under 2 years of age attended at a nutritionist's office at a municipal maternity hospital. R. pesq.: cuid. fundam. Online, [Teresina], v. 5, n. 6, p. 207216, dez. 2013;

CARVALHO, P.R.R.M. et al. Características e Segurança do Glutamato Monossódico como Aditivo Alimentar: Artigo de Revisão. Visão Acadêmica, Curitiba, v. 12, n. 01, p. 53-60, Jan./Jun. 2011;

CORREIA, P.P; PEREIRA, S.M.P.D; BRITO, L.A.M.H. Alimentação de transição infantil: conhecer para educar. Revista Ciência \& Saúde, Porto Alegre, v. 6, n. 2, p. 85-93, mai./ago. 2013;

DIAS, A.R. Alimentação Complementar De Crianças Menores De Dois Anos Residentes Na Vila Planalto, Brasília - DF. 38f. 2013. Trabalho de Conclusão de Curso (Graduação em Nutrição) - Universidade de Brasília, Brasília, 2013; 
FAVERO; D.M; RIBEIRO, C.S.G; AQUINO,

A.D. Sulfitos importância na indústria

alimentícia e seus possíveis malefícios à

população. Segurança Alimentar e

Nutricional, Campinas, v. 18, n. 1, p. 11-20, 2011;

FIGUEIRÓ, 1.S. Influência da redução do teor de nitrito de sódio na estabilidade oxidativa e avaliação microbiológica de linguiça suína frescal. 2013. 69f. Dissertação (Pós-graduação em Ciências da Tecnologia). Universidade Federal do Espirito Santo, Alegre, 2013;

FLORES, T.R. et al Consumo de refrigerantes entre escolares de séries iniciais da cidade de Pelotas, Rio Grande do Sul. Rev Cienc Saude, [Pelotas], v. 6, n.1, p. 59-66, Jan/Abr. 2013;

HEITOR, S.F.D; RODRIGUES, L.R;

SANTIAGO, L.B. Introdução de alimentos supérfluos no primeiro ano de vida e as repercussões nutricionais. Cienc. Cuid. Saúde, Campinas, v. 10, n. 3, p. 430-436, jul./set. 2011;

\section{HOCAYEN, P.A.S. Efeito da administração} oral do extrato de Baccharis dracunculifolia na obesidade induzida por glutamato monossódico (MSG), 2012. 108f. Dissertação (Mestrado em Ciências Biológicas) Universidade Estadual de Ponta Grossa,
Universidade Estadual do Centro Oeste, Ponta Grossa, 2012;

LEVY-COSTA, R.B., et al. Disponibilidade domiciliar de alimentos no Brasil: distribuição e evolução (1974-2003). Rev. Sa Pública, São Paulo, v.39 n.4, p. 530-540, Aug. 2005;

MARQUES, R.F.S.V. et al. Práticas inadequadas da alimentação complementar em lactentes, residentes em Belém-PA. Revista Paraense de Medicina, [Belém, PA], v. 27, n. 2, Não Paginado, abr./jun. 2013;

MARZANO, J. B. T. Fatores condicionantes para introdução precoce de alimentos industrializados em crianças de 4 meses a 5 anos de vida. 2013. $16 \mathrm{f}$. Monografia (Bacharelado em Nutrição)— Universidade de Brasília, Brasília, 2013;

MONTE, C.M.G; GIUGLIANI, E.R.J. Recomendações para alimentação complementar da criança em aleitamento materno. Jornal de Pediatria, [Rio de Janeiro], v. 80, n. 5, p.131-141, 2004;

OLIVEIRA, E; BOSCO, S.M. Hábitos alimentares de crianças com dois anos de idade em escolas municipais da cidade de lajeado, RS. Revista Destaques Acadêmicos, [S.1.], v. 1, n. 3, p. 1-9, 2009; 
OLIVEIRA, M.A.A; OSÓRIO, M.M.

Consumo de leite de vaca e anemia ferropriva na infância. J Pediatr, [RJ], v. 81, n. 5, p. 361367, 2005;

PEREIRA, A. C. S.; MOURA, S. M.;

CONSTANT, P. B. L. Alergia alimentar: sistema imunológico e principais alimentos envolvidos. Semina: Ciências Biológicas e da Saúde, Londrina, v. 29, n. 2, p. 189-200, jul./dez. 2008;

\section{PILLA, C. S. Perfil das denúncias recebidas} pelo Programa de Alimentos da Vigilância Sanitária de Viamão/RS. 2009. 45f.

Monografia (Graduação em Medicina Veterinária) - Universidade Federal do Rio Grande do Sul - Faculdade de Medicina Veterinária, Porto Alegre, 2009;

POLÔNIO, M. L. T. Percepção de mães quanto aos riscos à saúde de seus filhos em relação ao consumo de aditivos alimentares: o caso dos pré-escolares do Município de Mesquita. 2010. 129f. Tese (Doutorado em Ciências na área de Saúde Pública e Meio Ambiente). Escola Nacional de Saúde Pública Sergio Arouca, Rio de Janeiro, 2010;

\section{SEMEDO, J. Aditivos Alimentares em Cabo}

Verde. 2009. 57f. Monografia (Licenciatura em Ensino da Química), Departamento de Ciências e Tecnologia - Universidade de Cabo Verde, Cabo Verde, 2009;
SINON, V.G.N; SOUZA, J.M.P; SOUZA,

S.B. Aleitamento materno, alimentação complementar, sobrepeso e obesidade em préescolares. Rev. Saúde Pública, São

Paulo, v.43 n.1, fev. 2009;

\section{TEIXEIRA, A.R.N. Alergias Alimentares na}

Infância. 2010. 43f. Monografia (Graduação em Nutrição) - Faculdade de Ciências da Nutrição e Alimentação - Universidade do Porto, Porto, 2010.

TOLONI, M.H.A. et al. Introdução de alimentos industrializados e de alimentos de uso tradicional na dieta de crianças de creches públicas no município de São Paulo. Revista de Nutrição, Campinas, v. 24, n. 1, p. 61-70, jan./feb. 2011;

VINHOLES, D.B; ASSUNÇÃO, M.C.F; NEUTZLING, M.B. Freqüência de hábitos saudáveis de alimentação medidos a partir dos 10 Passos da Alimentação Saudável do Ministério da Saúde. Pelotas, Rio Grande do Sul, Brasil. Cad. Saúde Pública, Rio de Janeiro, v. 25 n.4, p.791-799, abr. 2009;

VITOLO, M.R et al. Consumo precoce de alimentos não recomendados por lactentes do sul do Brasil. Revista Ciência \& Saúde, Porto Alegre, v. 6, n. 1, p. 25-28, jan./abr., 2013; 
WHO (Organização Mundial de Saúde); WHA

Resolution 54.2. Geneva (SW): World Health

Organization; 2001. 